\title{
DIE BEENDIGUNG DER KONFESSIONALISIERUNG DER PRAGER UNIVERSITÄT AN DER WENDE VON DEN 1450ER ZU DEN 1460ER JAHREN AM BEISPIEL DES SCHICKSALS AKTIVER KATHOLISCHER MAGISTER
}

\author{
BLANKA ZILYNSKÁ
}

\section{CULMINATION OF THE CONFESSIONALIZATION OF THE UNIVERSITY OF PRAGUE AT THE END OF THE 1450S AND BEGINNING OF THE 1460S IN THE EXAMPLE OF THE FATE OF ACTIVE CATHOLIC MASTERS}

\begin{abstract}
The paper focuses on a group of masters loyal to the Catholic administrator Hilarius Litoměřický who attempted to take control of the university at the end of the 1450s. Their gradual removal from the ranks of members of the University of Prague concluded the search for the school's profile. By 1462, it had become a well-defined Utraquist institution linked to the Church through communion under both kinds. The episode is an example of the politization of the activities of masters, particularly in academic self-administration.
\end{abstract}

Keywords: University of Prague - fifteenth century - confessionalization - Utraquism - Hilarius Litoměřický - Wenceslaus Křižanovský

DOI: $10.14712 / 23365730.2020 .19$

Nach dem Ende der Hussitenkriege nahm die Prager Universität ihre Tätigkeit wieder auf. Statt von einer Universität sollten wir aber besser von der Prager Artistenfakultät sprechen, die als einzige völlig zu neuem Leben erwachte. Während der Revolution, als der Studienbetrieb an der Hohen Schule eingestellt war, engagierten sich die übrigen Magister für die Hussiten. Sie hielten Disputationen über das Dogma ab, und zwar sowohl mit der katholischen Partei als auch mit den radikalen Taboriten. Die Universität nahm man als bedeutenden politischen Akteur wahr, der zugunsten des Laienkelchs agierte. ${ }^{1}$

Es ist also kein Wunder, dass die Hohe Schule in den ersten Jahren nach Wiederaufnahme des Lehrbetriebs ausschließlich von Studenten aus den böhmischen Ländern frequentiert

1 František Šmahel, Die Prager Universität und der Hussitismus, in: Alexander Patschovsky - Horst Rabe (Hgg.), Die Universität in Alteuropa, Konstanz 1994, S. 111-128, Neudruck in: Ders., Die Prager Universität im Mittelalter. Gesammelte Aufsätze / The Charles University in the Middle Ages. Selected Studies (Education and Society in the Middle Ages and Renaissance 28), Leiden - Boston 2007, S. 172-195; Jiří KeJǨ, Mistři pražské univerzity a kněži táboršti [Die Magister der Prager Universität und die Priester der Taboriten], Praha 1981; Howard Kaminsky, The University of Prague in the Hussite Revolution: The Role of Masters, in: John W. Baldwin - Richard A. Goldthwaite (eds.), Universities in Politics, Baltimore - London 1972, S. 79-106; František Michálek BARToš, Z dějin Karlovy university v době husitské [Aus der Geschichte der Karlsuniversität zur Zeit der Hussiten], Jihočeský sborník historický 40, 1971, S. 54-63, 115-122. 
wurde. Bald jedoch wurde ihr Ruf besser und in den 1440er Jahren begannen sich die Hörsäle sowie die Magister- und Studentenkollegien zu füllen. Es begann eine kurze Zwischenzeit, in welcher die Universität auch ausländische Interessenten begrüßen konnte. Die ersten Ausländer kamen 1443 aus Wien, wo es nach einem Streit zu einer Trennung gekommen war. Die Zuwanderung von ausländischen Akademikern war ein erster Durchbruch im utraquistischen Monopol der Prager Schule. ${ }^{2}$

Zwar stimmte der Stadtrat der Aufnahme ausländischer Akademiker zu, doch war es gerade das städtische Milieu, das Anlass zu den ersten Vorfällen mit den ausländischen deutschen Katholiken gab. Die langsame Erneuerung der alten Verhältnisse war also gegen den Geist zunächst der Prager Stadtbürger. Die böhmischen Magister schienen sich der erstarkenden Konkurrenz und der drohenden Rekatholisierung anfangs nicht bewusst zu sein. Diesen Problemen brach dann die politische Entwicklung die Spitze ab: Als Georg von Podiebrad Anfang September 1448 Prag beherrschte, verließen die ausländischen Hörer und Magister lieber die Stadt. Sie fürchteten eine Rückkehr der revolutionär-hussitischen Verhältnisse. Die Universität wurde abermals eine Schule nur für einheimische Interessenten, eine Landesuniversität, wenngleich konfessionell noch nicht festgelegt. ${ }^{3}$

Die politische und konfessionelle Profilierung der Hohen Schule war aber noch längst nicht beendet. Die entscheidende Zeit waren die 1450er Jahre. Zwei Faktoren spielten hier eine Rolle: die Thronbesteigung des katholischen Königs Ladislaus Postumus und die Anwesenheit einer neuen Magister-Generation in den Universitätshörsälen. Ladislaus verhehlte nicht seine Unterstützung für die katholischen Kreise im Lande, die dadurch an Selbstbewusstsein gewannen. ${ }^{4}$ Und die Gruppe jüngerer Magister, die von ihren Auslandsstudien zurückkehrte, hatte auch neue Erfahrungen aus dem katholischen Europa, insbesondere aus Italien, und neue Vorstellungen im Gepäck, wie die Verhältnisse im Lande zu ordnen seien. Deshalb strebte die Lage an der Universität auf einen Konflikt und auf eine entschiedene Kollision zu.

2 František ŠMahel, Počátky humanismu na pražské universitě v době poděbradské [Die Anfänge des Humanismus an der Prager Universität zur Zeit Georgs von Podiebrad], Acta Universitatis Carolinae - Historia Universitatis Carolinae Pragensis (weiter AUC-HUCP) 1, 1960, S. 55-90, Neudruck in: Ders., Alma mater Pragensis, Praha 2016, S. 527-549; František ŠmAHEL - Miroslav Truc, Studie k dějinám University Karlovy v letech 1433-1622 [Studien zur Geschichte der Karlsuniversität 1433-1622], AUC-HUCP 4/2, 1963, S. 3-59, Neudruck in: F. Šmahel, Alma mater Pragensis, S. 411-458; Pavel KoTAu, Mistři pražské artistické fakulty v letech 1437-1448 [Die Magister der Artistenfakultät 1437-1448], Diplomarbeit an der Philosophischen Fakultät der Karlsuniversität, Praha 2011; Michal Svatoš, Kališnická univerzita (1419-1556) [Die utraquistische Universität (1419-1556)], in: Ders. (ed.), Dějiny Univerzity Karlovy, I, 1347/48-1622, Praha 1995, S. 205-226, bes. S. 207-208.

3 Eine Episode mit deutschen Studenten haben die Alten böhmischen Annalen verzeichnet, Zitat bei F. S̆MAHEL, Počátky humanismu na pražské universitě, S. 57-60. Zur Konfessionalisierung auf theoretischer Ebene und zur Anpassung an das böhmische Milieu Martin NodL, Konfessionalisierung und religiöse (In)Toleranz in Prag in der zweiten Hälfte des 15. Jahrhunderts, Bohemia. Zeitschrift für Geschichte und Kultur der böhmischen Länder 58, 2018, S. 286-309, kurz zur Prager Universität S. 291. Vgl. weiter Jan HrdinA - Kateřina JišovÁ, Die Koexistenz zweier Konfessionen in Prag 1436-ca. 1520, in: Michael Diefenbacher - Olga Fejtová - Zdzislaw Noga (Hgg.), Krakau - Nürnberg - Prag. Stadt und Reformation. Krakau, Nürnberg und Prag 1500-1618 (Documenta Pragensia - Monographia 33/2), Praha - Červený Kostelec 2019, s. 65-88.

4 David Papají, Ladislav Pohrobek (1440-1457). Uherský a český král [Ladislaus Postumus (1440-1457). Ungarischer und böhmischer König], České Budějovice 2016; Petr Čonnej, Ladislav Pohrobek, in: Marie Ryantová - Petr Vorel (edd.), Čeští králové [Die böhmischen Könige], Praha - Litomyšl 2008, S. $251-261$. 
Zu den aktiven Lehrern der Artistenfakultät gehörte auch der gewählte Prager Erzbischof, der höchste Vertreter der Utraquisten, Magister Johannes Rokycana. Sein Ziel war es, der Kirche genügend priesterlichen Nachwuchs und der Universität gut ausgebildete Lehrer zu sichern, und zwar auch für die juristischen und theologischen Fächer. Deshalb entsandte er seine fähigen Absolventen ins Ausland, damit sie dort die Weihen erhielten und an den dortigen Universitäten die entsprechenden Kenntnisse und akademischen Grade erwarben. ${ }^{5}$

Bei den weiteren Ereignissen spielten vor allem zwei der so entsandten Studenten eine Rolle: Hilarius von Leitmeritz und Wenzel von Křižanov (Křižanovský). Nach seinen Studien in Prag begab sich Hilarius mit Unterstützung utraquistischer Institutionen zu einem Studienaufenthalt nach Italien. Nach seiner Rückkehr trat er zwar eine Anstellung an der Universität an, doch bald darauf konvertierte er und wechselte auf bedeutende katholische Kirchenposten über. ${ }^{6} \mathrm{Ab}$ Jahresende 1461 war er schließlich katholischer Administrator. Von diesen Posten aus griff er auch in das Geschehen der Universität ein, während sein Freund, der agile Wenzel Křižanovský, direkt an der Universität an der Spitze der Katholiken stand.7 Diese beiden Männer waren die Hauptakteure der katholischen Opposition gegen den utraquistischen Kern der Universitätsmitglieder.

II.

Das Schauspiel ${ }^{8}$ begann am 11. März 1456 unter dem Dekanat von Stanislaus von Welwarn. ${ }^{9}$ Impuls war die Promotion von Bakkalaurei, welche im Geist der utraquistischen

5 Zu Rokycanas Absichten bezüglich der Universität und den gegenseitigen Beziehungen bspw. Zdeněk NEJEDLÝ, Mládí M. Jana z Rokycan [Die Jugend des Magisters Johannes Rokycana], Časopis Musea Království českého 73, 1899, S. 517-534, bes. S. 523, 531; Tomáš Kalina, Václav Křižanovský, Český časopis historický 5, 1899 , S. 333-359, hier S. 338-339; Rudolf URBÁNeK, Věk poděbradský, IV, Čechy za kralování Jiř́ki z Podébrad. Léta 1460-1464 [Das Podiebradsche Zeitalter, IV, Böhmen unter Georg von Podiebrad. Die Jahre 1460-1464] (České dějiny III/4), Praha 1962, S. 238, 241 Anm. 23, und passim.

6 Ota PAVLiČEK, Hilarius Litoměřický († 1468), teolog, univerzitni profesor a administrátor katolické konsistoře [Hilarius von Leitmeritz ( $\uparrow 1468)$, Theologe, Universitätsprofessor und Administrator des katholischen Konsistoriums], in: Petr Hlaváček et alt., Kacířská univerzita. Osobnosti pražské utrakvistické univerzity 1417-1622, Praha 2013, S. 57-61; Jaroslav KADLEC, Hilarius Litoměrický v čele duchovenstva podjednou [Hilarius von Leitmeritz an der Spitze der Geistlichkeit unter einer Gestalt], in: In memoriam Josefa Macka, Praha 1996, S. 187-196; Tomáš Kalina, Hilarius Litoměřický, Český časopis historický 5, 1899, S. 311-321; Ders., Václav Křžăanovský, 1. c. In der Literatur gab es Zweifel, ob seine Studienreise direkt von Johannes Rokycana finanziert worden sei, s. R. URBÁnek, Věk poděbradský, IV, S. 238-239.

7 T. Kalina, Václav Křż̌anovský, 1. c.; Eva GrEGorovičovÁ, Soukromé knihovny mistrů kolem pražského vysokého učení v pohusitském období [Die Privatbibliotheken der Prager Universitätsmagister in nachhussitischer Zeit], Doktorarbeit an der Philosophischen Fakultät der Karlsuniversität, Praha 1976, hier zu Křižanovský S. 65-123.

8 Zum Streit und den Folgeereignissen am eingehendsten T. Kalina, Václav Křižanovský, S. 334-351, bes. S. 335 a 341, und R. URBÁneK, Věk poděbradský, III, (České dějiny III/3), Praha 1930, S. 88-89, IV, S. 236249; ältere Arbeiten verzeichnet Ders., Bd. IV, S. 236, Anm. 11 (B. Balbín, A. Voigt, V. V. Tomek). Vgl. auch František Kavka (Red.), Stručné dějiny University Karlovy [Kurze Geschichte der Karlsuniversität], Praha 1964, S. 77-78 (Zusammenfassung und Auswertung der Ereignisse durch F. Šmahel mit anderen als den hier im Text angeführten Akzenten); kurz auch F. ŠMaHeL, Počátky humanismu na pražské universitě, S. 61-62; faktographisch ungenau M. Svatoš, Kališnická univerzita, S. 208-209.

9 Über ihn Eva Gregorovičové, Knihovna mistra Stanislava z Velvar [Die Bibliothek des Stanislaus von Welwarn], AUC-HUCP 18/1, 1978, S. 107-127. 
Lehre es ablehnten, den Eid abzulegen. ${ }^{10}$ Sie verwiesen auf die Matrikel des Rektors, in welcher die Aufzeichnung der Statuten ein ,iurare aut promittere“ (,,schwören oder geloben") einräumte. Der Dekan verwies demgegenüber auf die im Fakultätsbuch verzeichneten Statuten, in dem nur das Wort ,, iurare " stand. In diesem ersten Akt gewannen die Anwärter auf den Bakkalaureus-Grad: diesmal gestattete der Dekan nach Beratung mit dem Rektor, um Zwistigkeiten zu vermeiden, dass sie sich die Form des Gelöbnisses aussuchen konnten. Über alles weitere sollte eine Versammlung aller Magister entscheiden. ${ }^{11}$

Ein Problem entstand auch bei der Entscheidung über den Aufnahmeantrag von 6 neuen Magistern in den Fakultätsrat. Zwar war dies nur ein Streit über das formale Vorgehen, doch diente er zugleich als Kraftprobe. Unter den Antragstellern waren am 1. April 1456 auch Wenzel Křižanovský und Johannes Krčín. Dekan Stanislaus von Welwarn entschied zugunsten jener, die das obligatorische Biennium bereits erfüllt hatten. Rektor Martin von Lenčice (de Lancicia) stimmte mit seinem Rat der Aufnahme am 22. 4. zu, wie am selben Tag auch die Fakultätsversammlung. Allerdings fügt der Schreiber hinzu, dass dies viele Schwierigkeiten zur Folge hatte. ${ }^{12}$ Kř̌žanovský (28jährig), der dem geforderten Biennium entsprach, wurde in den Rat aufgenommen und tags darauf für den nächsten Zeitraum zum Dekan der Fakultät gewählt. ${ }^{13}$

In seiner Amtszeit kehrte das Fakultätskollegium zur Frage des Gelübdes zurück. Für die Bakkalaurei blieb das ,,iurare "verpflichtend, und für die Magister wurde zunächst (4. 2. 1457) das Gleiche angeordnet. ${ }^{14}$ Dann zeigte es sich allerdings, dass Křržanovský als Dekan das Wort ,promittet “ (,er wird geloben“) in den Statuten zu ,,iurat “ (,,er schwört") in dem die Magister betreffenden Artikel korrigiert hatte, ohne dies mit der Fakultät besprochen zu haben..$^{15}$ Er selbst war unterdessen (nach dem 5. 5. 1457) zu Studien nach Italien abgereist. Die Angelegenheit musste erst von Dekan Hieronymus von Skutecz geklärt

10 Nach F. Šmahel, Počátky humanismu na pražské universitě, S. 61, wurde der Schwur auf die Kompaktaten geleistet, doch spricht die Quelle (s. weiter) nur von einem Schwur als üblichen Teil der Promotion. In diesem Sinne R. URBÁNEK, Věk poděbradský III, S. 88-89. Die Vorgeschichte der Auseinandersetzung um den Schwur auf dem Boden der Universität hat anhand von diesbezüglichen Fällen aus den Jahren 1414-1418 Martin NoDL, Iurare aut promittere. Př́spěvek $k$ problematice pražských univerzitních statut [Iurare aut promittere. Ein Beitrag zur Problematik der Prager Universitätsstatuten], AUC-HUCP 47/1-2, 2007, S. 49-57, aufgespürt. Vgl. auch F. Šmahel, Nejstarši statuta Univerzity Karlovy [Die ältesten Statuten der Karlsuniversität], in: František Šmahel - Gabriel Silagi (edd.), Statuta et acta rectorum universitatis Carolinae Pragensis 1360-1614 (Documenta Historica Universitatis Carolinae Pragensis I), Praha 2018, S. XXXIV-XXXVI, wo auch die Auseinandersetzung um den Schwur 1409 und daran anschließend 1456 reflektiert wird.

11 Die Situation beschrieb der Dekan Stanislaus von Welwarn in dem amtlichen Dekansbuch: Liber decanorum, Monumenta historica Universitatis Karolo-Ferdinandeae Pragensis (weiter MUP), I/2, Pragae 1830, S. 53-54.

12 MUP I/2, S. 53 (Aufnahmeantrag), S. 55 (Beschluss des Rektors und der Fakultätsversammlung). Bei der Einberufung der Versammlung Anfang April wurde nicht angegeben, dass über die Aufnahme der Magister verhandelt werden soll, weshalb Unstimmigkeiten entstanden. Der Schreiber kommentiert die Entscheidung wie folgt: „Et hoc facto, conclusio in principio ad petitionem eorum, decano consentiente uni parti, firmitate perhibetur roborata juxta consuetudinem et praxim tunc observatam a spatio annorum quam plurium; habuit enim haec conclusio multas difficultates cupientium eam infringere." (ibidem, S. 55-56). T. KaLinA, Václav Křižanovský, S. 334.

13 MUP I/2, S. 56. Die Dekanswahl wurde um eine Woche verschoben und fand erst am 23. 4. statt, weil man die Entscheidung des Rektors abwartete.

14 MUP I/2, S. 57-58: Beschluss der Fakultätskonvokation; T. Kalina, Václav Křižanovský, S. 335; R. URBÁNEK, Věk poděbradský III, S. 88-89.

15 Nach F. Šmahel, Nejstarši statuta Univerzity Karlovy, S. XXXVI, wurde Křižanovský diese Statutenkorrektur von Rektor Stanislaus von Welwarn genehmigt. 
werden, der sich an Rektor Johannes von Jemnitz wandte. ${ }^{16}$ Unter seiner Aufsicht entschied die Magisterkongregation am 27. 6. 1458, dass die Magistranden nur geloben werden - ,promittere“-, wie dies nach Erinnerung der älteren Magister ursprünglich der Brauch war. ${ }^{17}$ Der Beschluss wurde vom Dekan auf ein Blatt mit den Aufzeichnungen seiner Amtszeit notiert und fand Eingang in den Wortaut der Statutenabschrift in demselben Dekansbuch. ${ }^{18}$

In derselben Versammlung wurde auch die Frage der Wahrung der Kelchkommunion durch die Universität behandelt. Man fasste den Beschluss, die Universitätsangehörigen hätten sich an die ,littere universitatis recomendantis communionem utriusque speciei ad vulgus" zu halten - an den Universitätsbeschluss, der die Kommunion für Laien unter beiderlei Gestalt empfahl: offensichtlich handelte es sich um die Entscheidung vom 10. März $1417 . .^{19}$

Im Dekansbuch sind drei scheinbar ungleichartige Episoden vermerkt: die Krönung König Georgs, die Durchsetzung des utraquistischen Standpunkts zum Gelübde und die Pflicht zur Verteidigung des Kelchs. ${ }^{20}$ In Wirklichkeit sind diese Episoden aber innerlich miteinander verflochten. Die Regierungszeit von Ladislaus Postumus bot der katholischen Gruppe eine Chance, was sich in dem Gewinn guter Magisterpositionen bspw. bei Wenzel Křižanovský und Stanislaus von Welwarn zeigte oder darin, wie die Forderungen der katholischen Herren, die sie an Georg vor seiner Krönung stellten, formuliert sind: da taucht unter anderem das Verlangen nach Gleichheit der katholischen Magister und Studenten an

16 MUP I/2, S. 64-65 (in der Handschrift fol. 142r). Am detailliertesten T. KaLinA, Václav Křrižanovský, S. 335-336, 340-341.

17 T. Kalina, Václav Křžăanovský, S. 340.

18 MUP I/1, S. 58-60, Nr. 30 und 32; in Faksimile-Ausgabe Karel BeráneK - J. V. ČErnÝ - Marie Pravdová (edd.), Liber decanorum facultatis philosophicae Universitatis Pragensis ab anno Christi 1367 usque ad annum 1585, Pragae 1983, S. 38-39 (Handschrift fol. 18v und 19r), F. ŠMAHEL - G. Silagi, Statuta, IV/3: Statuta facultatis artium..., S. 227-275, hier S. 244-245. Artikel 30 benutzt das Wort ,promittet“, ohne dass hier eine Rasur sichtbar wäre - es handelt sich also um eine Abschrift einer korrigierten Vorlage, am ehesten aus der Rektorenmatrikel, auf die in der Niederschrift auf fol. 142r. verwiesen wird. Nach Artikel 32 folgt ein Zusatz, der mittels Strich mit Artikel 30 verbunden ist, auf welchen er sich bezieht und wo sich der Hinweis findet: „Vide plus in alia parte litere“. Der Zusatz erklärt, dass die Art des Schwurs von ,jurabit“ zu ,,promittet“" korrigiert wurde, weil dies nach den Erinnerungen älterer Magister ursprünglich so gewesen war. Unverändert blieb der 13. Artikel der II. Rubrik der Fakultätsstatuten (MUP I/1, S. 49-51, Nr. 13; Ms. Fol. 16r; F. ŠMAHEL G. Silagi, Statuta, S. 240), überschrieben mit „Quae jurare debent post praesentationem“, der den eigentlichen Schwur nicht behandelt. Vgl. F. ŠMAHEL, Nejstarši statuta Univerzity Karlovy, S. XXXVI, XXXVIII-XXXIX.

19 Jiří KeJŘ, Deklarace pražské university z 10. března 1417 o přjímáni pod oboji a jeji historické pozadi [Deklaration der Prager Universität vom 10. März 1417 über die Kommunion unter beiderlei Gestalt und ihren historischen Hintergrund], Sborník historický 8, 1961, S. 133-156. Václav Vladivoj Tomeк, Dějepis města Prahy [Geschichte der Stadt Prag], IX, Praha 1893, S. 189-190, und nach ihm T. KaLinA, Václav Kř́žanovský, s. 342, auch R. UrBáNEK, Věk poděbradský IV, S. 236, identifizieren den Hinweis als Beschluss der Universität vom 1. 8. 1420, dass alle Magister zur Verteidigung der Vier Artikel verpflichtet sind. Dazu V. V. Tomek, Dějepis města Prahy, IV, Praha 1879, S. 93; Ders., Geschichte der Prager Universität, Prag 1849, S. 143, brachte den Beschluss noch mit der Anordnung von 1417 in Verbindung, ebenso Miroslav BoHÁčEK, Pražská universitní statuta a jejich boloňský vzor [Die Prager Universitätsstatuten und ihr Bologneser Vorbild], Studie o rukopisech 8, 1969, S. 11-64. Die Identifikation mit dem Dokument aus dem Jahr 1417 approbiert die Aufzeichnung in Statuta officii rectoratus (F. Šmahel - G. Silagi, Statuta, S. 202, Art. XXVI), vgl. F. Šmahel, Nejstarši statuta Univerzity Karlovy, S. XXXIX, Anm. 99.

20 Die beiden zuletzt Genannten hatten wir schon erwähnt, die Aufzeichnung über die Krönung und auch über den Aufstieg Viktorinus' zum Fürsten im Liber decanorum (MUP I/2, S. 59-60) fertigte Johannes von Prag als Nachtrag auf Folio 140v, wo es in der rechten Kolumne unter den Vermerken von Dekan Křižanovský eine freie Stelle gab, unter ausdrücklicher Namensnennung des Schreibers an. 
der Universität auf. ${ }^{21}$ Nach dem Amtsantritt Georgs wendete sich die Lage freilich. (Nicht nur) deshalb reflektierte die Universität bzw. die Dekane in ihrem Gedächtnisbuch wichtige Ereignisse in der Herrscherfamilie.

Also auch der zweite Akt dieses Schauspiels fiel zugunsten der utraquistischen Mehrheit aus. Weitere Schritte ließen aber nicht lange auf sich warten.

Als Křžzanovský als Doktor der Theologie von seinen italienischen Studien zurückgekehrt war, ersuchte er im Dezember ${ }^{22}$ gemeinsam mit seinem Kollegen Johann Krčín, Doktor der Medizin, um Aufnahme als Doktor auf die Prager Universität. Ihre Aufnahme bedingten die Magister mit dem Beitritt zum Fakultätsbeschluss vom Juni 1458, welcher die Kelchkommunion betraf.

Diesen Beschluss bestärkten die Kollegiaten des Karolinums noch am 13. Dezember 1459 mit der Entscheidung, nur denjenigen unter sich aufzunehmen, der sich zur Kommunion unter beiderlei Gestalt verpflichte. ${ }^{23}$ Dies bezog sich wohl auch auf das Allerheiligenkolleg, wo Křižanovský seinen Platz hatte. Da Křižanovský in Italien bereits konvertiert war, wollte er sich den Forderungen nicht fügen. Deshalb wurden ihm Handlungen verwehrt, die mit dem Recht zusammenhingen, Absolventen zu prüfen und zu promovieren. Er geriet somit mit den Magistern des Karolinums in einen langen Streit und bildete zusammen mit anderen an der Fakultät eine Opposition, die sich bereits im Frühjahr 1460 äußerte.

III.

Am 16. April 1460 wurde Wenzel Koranda junior auf Wunsch von Johannes Rokycana zum Dekan gewählt. ${ }^{24}$ Gegen den irregulären Wahlverlauf erhob Johannes Krawin von Tabor Einwände, weitere sechs Magistern verweigerten Koranda das Gehorsamsgelübde. ${ }^{25}$

21 Über die Forderungen V. V. Tomek, Dějepis města Prahy, VI, Praha 1885, S. 305; T. Kalina, Václav Křižanovský, s. 342; R. URBÁNEK, Věk poděbradský III, S. 347-353, über die Universität S. 351-352. Die Anfang Mai 1458 datierten Forderungen sind von Adolf Bachmann, Urkunden und Actenstücke zur österreichischen Geschichte im Zeitalter Kaiser Friedrichs III. und König Georgs von Böhmen (1440-1471), Fontes rerum Austriacarum, II. Abt., Diplomataria et acta, Bd. 42, Wien 1879, S. 237-241, Nr. 170 herausgegeben, über die Universität S. 241 (zeitgenössische deutsche Übersetzung aus einer Weimarer Handschrift), eine kürzere tschechische Fassung in der Handschrift Stuttgart, Theol. Philos. Q Nr. 37, f. 276v-277v.

22 Der Eintrag im Dekansbuch (MUP I/2, S. 70-71) ist keinem Jahr eindeutig zugeordnet: die Datierung, Freitag trockener Dezembertage“ könnte der 19. 12. 1460 oder der 21. 12. 1459 sein. T. KaLina, Václav Křižanovský, S. 343, Anm. 1, erklärt, warum er sich für das Jahr 1459 entschied, als Tag führt er allerdings den 19. 12. an; F. ŠMahel, Počátky humanismu na pražské universitě, S. 61, Anm. 31, spricht von der Datierung des Eintrags zum 9. 12. 1460 und hält dies für einen Fehler im LD, das Ereignis soll ins Jahr 1459 gehören.

23 Statuta collegii Caroli Quarti, in: F. Šmahel - G. Silagi, Statuta, S. 285-293, hier S. 291-292, § 17, Kommentar auf S. LX; T. Kalina, Václav Křižanovský, S. 342; Václav Vladivoj Tomek, Paměti kollegiatů kolleje Karlovy [Erinnerungen der Kollegiaten des Karlskollegs], Časopis Českého museum 21/1, 1847, S. 382 passim 646, hier S. 533-534 (tschechische Übersetzung des Beschlusses); Ders., Dějepis města Prahy, IX, s. 190; Tomek und nach ihm Kalina führen als Datum irrtümlich den 10. 12. 1459 an, obgleich das LD den 13. 12. nennt.

24 MUP I/2, S. 73, 75-76. Eine neue Biographie hat Jindřich MAREK, Václav Koranda mladši. Utrakvistický administrátor a literát [Václav Koranda der Jüngere. Utraquistischer Administrator und Literat], Praha 2017, herausgegeben, zu den Ereignissen nach der Wahl Korandas zum Dekan S. 22-23; ph (Petr Hlaváček), Václav Koranda ml. († 1519), in: Ders. et alt., Kacířská univerzita. Osobnosti pražské utrakvistické univerzity 1417-1622 [Die ketzerische Universität. Persönlichkeiten der Prager Utraquistenuniversität 1417-1622], Praha 2013, S. 62-64.

25 Dies waren: Stanislaus von Welwarn, Wenzel von Křižanov, Johann von Krčín, Nikolaus von Hořepník, Valentin von Rakowitz, der bereits genannte Johannes Krawin von Tabor und Georg aus der Prager Altstadt. 
Obwohl der König die Wahl bestätigte, ${ }^{26}$ ordnete sich die Opposition dem neuen Dekan nicht unter, stellte die Vorlesungen ein und beteiligte sich nicht an Disputationen. Wir werden verfolgen, wie sich die Fakultät ihrer entledigt.

Der Widerstand äußerte sich auch auf andere Weise. Křižanovský weigerte sich, bei einer Revision der Bibliothek im Kolleg der Böhmischen Nation ausgeliehene Bücher zurückzugeben, und versuchte die Debatte auf die Ebene eines juristischen Kompetenzstreits zu heben. Als die Angelegenheit dem Rektor vorgelegt wurde, erhielt dieser zur Antwort, dass er - Křrižanovský - Priester der Prager Kirche sei und der Rektor ihm gegenüber keine Rechtsbefugnis habe. Křižanovský legte beim Prager Kapitel Berufung ein und beschwerte sich bei Hilarius von Leitmeritz. ${ }^{27}$ Daraufhin verkündete der Rektor, Křižanovský sei kein Mitglied der Universität mehr. Letzterer schrieb dem Rektor einen Brief, der in die Statutenhandschrift eingefügt ist und in welchem er auch den Wunsch äußerte, aus der Matrikel gestrichen zu werden. Dies erfolgte dann irgendwann im Juli 1461. ${ }^{28}$ Křižanovský wurde also als erster aus der Universität ausgestoßen und musste auch das Allerheiligenkolleg verlassen. An seine Stelle kam Johann Krčín, der Leibarzt Georg von Podiebrads und zweiter der oppositionellen sechs Magister. Freilich blieb er nur kurze Zeit im Kolleg, da er 1464 heiratete. ${ }^{29}$ Er wurde von dem Utraquisten Wenzel Pala von Prag ersetzt. ${ }^{30}$

Ausgeschlossen wurden auch die Magister Stanislaus von Welwarn und Georg von Meziříčí, die ähnlich wie Křižanovský dem Rektor den Gehorsam aufkündigten. Vorwand für ihren Ausschluss war ihre Weigerung, sich 1461 zu Verhandlungen mit dem Rektor über finanzielle Angelegenheiten einzufinden, die unter Welwars Rektorat begonnen hatten. Als sie auch der zweiten Aufforderung nicht Folge leisteten, entschlossen sich die Magister,

Letzterer war frisch aufgenommener Magister-Regent (MUP I/2, s. 52, 54, 62, 63, 67), doch nach 1460 gibt es über ihn keinerlei Nachrichten - als einziger kommt er bei den kommenden Ereignissen nicht vor.

26 MUP I/2, s. 76.

27 T. Kalina, Václav Křižanovský, S. 345, und Ders., Hilarius Litoměřický, S. 313, fragt, warum sich Křižanovský an Hilarius wandte. Er erinnert daran, dass er wohl Offizial war und als Mitglied der königlichen Kanzlei Zugang zum König hatte. Spätestens seit 1459 aber war er Dekan des Allerheiligenkapitels und in dieser Funktion Konservator der Universitätsrechte. Vgl. ferner Anm. 34-36. Administrator war Hilarius erst ab Ende 1461. R. URBÁNEK, Věk poděbradský IV, S. 244, stimmt mit Kalina nicht darin überein, dass er Offizial bzw. Dekan des Veitskapitels gewesen sei.

28 Quelle sind die Rektoratsakten, in: Antonius DitTrich - Antonius SpIRK (edd.), Statuta Universitatis Pragensis nunc primum publici juris facta, MUP III, Pragae /1848/, S. 50 (Reprint F. ŠmaHEL - G. Silagi, Statuta, S. 54-55), wo die Auseinandersetzung um die Bücher sowie der Ausschluss beschrieben sind; eine tschechische Übersetzung des betreffenden Abschnitts bei V. V. ToмeK, Paměti kollegiati̊, S. 534-535. T. KaLINA, Václav Křižanovský, S. 344-346; František ŠmaHEL, Knihovni katalogy koleje Národa českého a koleje Rečkovy [Die Bibliothekskataloge des Kollegs der Böhmischen Nation und des Reček-Kollegs], AUC-HUCP 2/1, 1961, S. 59-85, hier S. 77-78 (Reprint: Ders., Die Bücherkataloge des Collegium Nationis Bohemiae und des Collegium Reczkonis, in: Ders., Die Prager Universität im Mittelalter, 1. c., Leiden - Boston 2007, S. 405-439, hier S. 430); Zuzana SilagiovÁ - František Šmahel (edd.), Catalogi librorum vetustissimi Universitatis Pragensis / Die ältesten Bücherkataloge der Prager Universität (Corpus Christianorum - Continuatio Mediaevalis 271: Magistri Iohannis Hus Opera omnia..., tomus XXXVIIB - Supplementum II), Turnhout 2015, S. XLIV und Anm. 101: Erwägung über die Identifizierung der Bücher, die Křižanovský nicht zurückgegeben hatte und die aus dem Nachlass Prokops von Pilsen stammten.

29 V. V. Tomek, Paměti kollegiati̊, S. 536; František ŠMahel, Humanismus v době poděbradské [Der Humanismus zur Zeit Georgs von Podiebrad], Praha 1963, S. 15-16 (Krčíns Studium in Bologna), S. 58 (seine Karriere); Gustav Gellner, Jan Černý a jini lékaři češti do konce doby jagellovské [Jan Černý und andere tschechische Ärzte bis zum Ende der Jagiellonenzeit], Věstník Královské české společnosti nauk 1934, č. III, S. 132-134 (Angaben über Krčíns Leben sind den Prager Stadtbüchern entnommen).

30 Als Mitglieder des Karlkollegs bzw. des Allerheiligenkollegs zum Jahr 1464 werden beide von V. V. TomeK, Paměti kollegiatü, S. 522 und 524, 536 ohne Quellenangabe genannt. 
beide nicht nur der Ausübung ihrer Pflichten zu entbinden, sondern sie auch aus der Universität auszuschließen. Stanislaus erhielt dann Pfründe im Prager Kapitel; Nachrichten über Georg fehlen. Im Kapitel endete auch Valentin von Rakowitz (Rakovice). Damit fielen also drei weitere Magister weg. ${ }^{31}$

Besonders demonstrativ rechnete man mit Johannes von Tabor, genannt Krawin ab: nach längerer Inhaftierung musste er am 17. 8. $1461 \mathrm{im}$ theologischen Lektorium des Karolinums die Verleumdungen Johannes Rokycanas, des Königs und der böhmischen Königin widerrufen, und zwar vor einem zahlreichen Publikum. Den Text des Widerrufs brachten zwei Vertraute des Königs mit: die Ritter Samuel von Hradek und Čeněk von Klinstein; der Verlauf wurde von drei Notaren notiert. ${ }^{32}$ Selbst der Widerruf verbesserte Krawins Ruf unter den Utraquisten nicht. Dies wird von einer Illustration in der Göttinger Handschrift bezeugt, in der Krawin in Opposition zu Johannes Rokycana und zu zwei utraquistischen Geistlichen steht: Zacheus und Wenzel Pala, welcher den Platz von Krčín im Allerheiligenkolleg erhielt. ${ }^{33}$

Der letzte Oppositionelle, dessen sich die Fakultät entledigte, war Nikolaus von Hořepník. Er wurde auf Geheiß des Königs wegen des Besitzes von Büchern des taboritischen Bischofs Nikolaus von Pilgram am 16. 4. 1461 verhaftet und inhaftiert. Der Rektor durchsuchte seine Kammer im Allerheiligenkolleg und fand dort die Bücher. Hořepník war ein Prager Kanoniker, also ein Katholik (er war freilich ein zum Katholizismus konvertierter Utraquist), so dass es sich hierbei nicht um Sympathie für die Taboriten handelte, sondern vielmehr um Studienmaterial zwecks antitaboritischer Polemik. Wie aus einem Zettel, den Hilarius an Hořepník geschrieben hatte und der als Beweisstück in das Statutenbuch geklebt war, hervorgeht, hatten Hořepník und Hilarius miteinander konspiriert. Auf dem Zettel teilt

31 Nach T. Kalina, Václav Křžăanovský, S. 348-349, seien nur Křižanovský und Hořepník ausgeschlossen worden, die Übrigen hätten bei Verhandlungen Stimmenthaltung üben müssen. Erst nach Aufhebung der Kompaktaten seien sie weggegangen. Die Aufzeichnung in den Rektorakten enthält jedoch die angegebenen Tatsachen, siehe MUP III, S. 58 (F. Šmahel - G. Silagi, Statuta, S. 60-61). Vgl. auch E. Gregorovičová, Knihovna mistra Stanislava, S. 112; R. URBÁNeK, Věk poděbradský, IV, S. 243, 246 Anm. 43, 247-248 Anm. 46. Ein Teil der Ausgeschlossenen fand Versorgung als Mitglieder des Kapitels, also bei Hilarius; Mutmaßungen der älteren Literatur (vgl. T. KALINA, Václav Křižanovský, S. 349-350) überprüfte Antonín MAŘiK, Svatovitská kapitula za vlády Jiřiho z Poděbrad [Das St.Veitskapitel unter der Herrschaft Georgs von Podiebrad], Documenta Pragensia 20, 2002, S. 25-53, und bestätigte die Zugehörigkeit zum Kapitel bei Wenzel von Rakowitz (Nr. 38), Nikolaus Hořepník (Nr. 41), Stanislaus von Welwarn (Nr. 49) und Wenzel Křižanovský (Nr. 54). F. ŠMAhel, Počátky humanismu na pražské universitě, S. 62, Anm. 36, führt darüber hinaus Georg von Meziřččí an, den aber A. Mařík in seinen Verzeichnissen nicht nennt. Georg figuriert bei Prüfungen zuletzt 1454 (MUP I/2, S. 48-50). Er taucht dann erst wieder im Zusammenhang mit einer Finanzaffäre auf. An dem Protest gegen Koranda beteiligte er sich nicht, es handelte sich hier um einen anderen Magister namens Georg (siehe Anm. 25).

32 MUP III, S. 55, Nr. 18, und S. 235-238; F. Šmahel - G. Silagi, Statuta, S. 58, Nr. 18, und S. 184-185, Informationen über die Form der Aufzeichnung dieses Ereignisses auf S. XXV-XXVI. Der Text des Widerrufs findet sich auf der hinteren Deckelinnenseite der Handschrift XIV D 25 der Nationalbibliothek. Über Krawin V. V. Tomeк, Dějepis města Prahy, IX, S. 169; R. URBÁnek, Věk poděbradský, IV, S. 247-249.

33 Handschrift Göttingen, Universitätsbibliothek, Sign. $2^{\circ}$ Cod.Ms.theol. 182, fol. 30r, Entstehung um 1464. Vgl. Karel CHYTIL, Antikrist v naukách a uměni středověku a husitské obrazné antithese [Der Antichrist in Lehre und Kunst des Mittelalters und die hussitische bildhafte Antithese], Praha 1918, S. 160-161; Rudolf UrBáNEK, Starši obdoba rukopisu Jenského, rukopis Göttinský [Eine ältere Analogie zum Jenaer Kodex, die Göttinger Handschrift], in: Ders., Z husitského věku, Praha 1957, S. 262-274. In der jüngeren Handschrift aus Jena ist diese Szene durch andere, aktuellere Gestalten ersetzt, was beweist, dass es sich im Falle von Krawin um eine lebendige Reaktion auf zeitgenössisches Geschehen handelte. Siehe Kamil Boldan - Kamil BrodsKÝ (edd.), Jenský kodex. Faksimile a komentár [Der Jenaer Kodex. Faksimile und Kommentar], Praha 2009, Kommentar: S. 120-121, Faksimile: fol. 25r. 
Hilarius mit, dass er ihm einen Haftbefehl für den Probst von Allerheiligen, wohl Křižanovský, geschickt habe, den er entgegen der Kompetenz des Rektors in Haft nahm. Hořepník sollte dies an der Universität melden. ${ }^{34}$ Offensichtlich auf Hilarius berief sich Hořepník auch in seinem Streit mit den Mitgliedern des Allerheiligenkollegs. ${ }^{35}$ Die Berufung auf das St.Veitskapitel bzw. auf Hilarius als Dekan des Allerheiligenkapitels (welcher Konservator der Universitätsrechte war) ${ }^{36}$ und die Inhaftierung seinerseits sollten offenbar nach Jahren die faktische Rechtskraft des Dekans über die Mitglieder des Allerheiligenkapitels erneuern. Hilarius wollte das Kapitel mit seinen Prälaten erneut integrieren, er selbst schreibt: „,integritas et status collegii reformari possit“. ${ }^{37}$ Im Ergebnis wurde Magister Hořepník aus dem Kolleg und schließlich auch aus der Universität ausgeschlossen.

\section{IV.}

Mit dem Ausschluss von Nikolaus von Hořepník war die katholische Opposition vollständig aus der Universität entfernt. ${ }^{38}$ Damit endete der Kampf um zwei Konzeptionen der weiteren Universitätsentwicklung. Hierbei ging es nicht nur um das Schicksal mehrerer Magister oder um irgendwelche, fünfzehn Bücher betreffende Lappalien. In einigen Momenten betraf der Streit, den eine konfessionell festgelegte Universitätsminderheit mit der Mehrheit führte, wichtige Punkte der Jurisdiktion der Universität, ihrer Autonomie und ihrer Freiheiten.

Křižanovskýs Verhalten lief auf eine Bagatellisierung des von Papst Bonifazius IX. 1397 verliehenen Privilegiums hinaus, welches die Jurisdiktion des Rektors über die Universitätsangehörigen ungeachtet ihres Status als Kleriker oder als Angehörige eines anderen Rechtsbereiches anerkannte. Ein Teil der Rechtsbefugnis sollte sich auf das Kapitel beziehen beziehungsweise auf die katholischen Administratoren. Der amtierende Rektor des Jahres 1461, Johannes von Prag, hatte ein Gespür dafür, wohin die Situation tendierte, und schrieb in seine Akten: ,Item Hilarius cum magistris apostasis false interpretati sunt privilegia universitatis, rectorem a iurisdiccione, quam habet super supposita, privare volentes,

34 MUP III, S. 51 (F. Šmahel - G. Silagi, Statuta, S. 55). Den Fall analysiert T. Kalina, Václav Křižanovský, S. 347-348; vor ihm V. V. Tomek, Geschichte der Prager Universität, S. 144; Ders., Dějepis města Prahy, VII, Praha 1886, S. 22. Eine weitere Quelle, die über die Inhaftierung Hořepníks aussagt, führt R. URBÁNEK, Věk poděbradský, IV, S. 244-246, bes. Anm. 34 an.

35 MUP III, S. 54, Nr. 16; Gefangenschaft und Auffindung der Bücher ibidem, S. 57, Nr. 26 (F. ŠmaHEL - G. SiLAGI, Statuta, S. 58, Nr. 16, und S. 59, Nr. 26).

36 Jiří STočEs, Konzervátoři práv predhusitského pražského obecného učeni [Die Prager Universitätskonservatoren aus vorhussitischer Zeit], AUC-HUCP 45/1-2, 2005, S. 29-66, hier S. 38-39, 63 und 43-44, wo er auch die vorhussitischen Aktivitäten des Dekans (Blasius gen. Wolf / dictus Lupus) des Allerheiligenkapitels und seinen Schritt zur Stärkung des Einflusses als Konservator der Universitätsrechte im Jahre 1400 beschreibt, ähnlich Ders., Pražské univerzitni národy do roku 1409 [Die Prager Universitätsnationen bis 1409], Praha 2010, S. 121-126, bes. S. 125-126.

37 MUP III, S. 51 (F. Šmahel - G. Silagi, Statuta, S. 55). Das Blatt zitiert T. Kalina, Václav Křižanovský, S. 347-348, 359, Anm. 1. Bei diesem Blatt handelt es sich wie bei dem von Křižanovský um ein Autograph; beide Blätter sind in das Statutenbuch geklebt: Handschrift der Nationalbibliothek Prag, Sign. XIV D 25, f. 25 r.

38 Vgl. die Zusammenfassung der Aktion gegen die katholischen Magister in: F. ŠMAHEL, Počátky humanismu na pražské universitě, S. 62. 
et capitulo conferre eandem conantes. “39 Das Ringen darum, wer den Universitätskanzler bei Sedisvakanz des erzbischöflichen Stuhles vertritt, entschied die Universität und ihr Rektor dank der utraquistischen Mehrheit für sich; sie gewannen somit gegen das Kapitel und den Administrator.

Es drohte somit die Teilung der Universität in eine utraquistische und eine katholische. Dem katholischen Teil gelang es nicht, eine Verbindung der kirchlichen mit den universitären Organen durchzusetzen, was zum Ausschluss der katholischen Gruppe aus der Universität führte. Im Falle der Utraquisten hingegen gelang ein solches Modell. Nach dem Tod von Johannes Rokycana war die utraquistische Kirche bzw. ihr Konsistorium seit den 70er Jahren des 15. Jahrhunderts personell, lokal und ideell mit den Universitätsmagistern und ihrem Sitz im Karolinum auf ganz wesentliche Weise verknüpft. ${ }^{40}$

Ein weiteres Ziel der beschriebenen Ereignisse war möglicherweise die Beherrschung des Allerheiligenkollegs durch die katholischen Magister. Die Kollegiaten rekrutierten sich seit Karl IV. aus den Reihen der Magister des Karlskollegs, die während des hier beschriebenen Streits ausschließlich Utraquisten waren, während die Würdenträger des einstigen Allerheiligenkapitels - also Probst und Dekan - nach den Hussitenkriegen vom Prager Kapitel aus den Reihen der Katholiken eingesetzt wurden. ${ }^{41}$ Die Absicht der Oppositionsgruppe war also unzweifelhaft, erneut beide Teile: Kapitel und Kolleg, auf katholischer Grundlage zu vereinen. Dies war für sie sowohl hinsichtlich des Prestiges als auch in wirtschaftlicher Hinsicht wichtig.

Das Jahr 1461 bedeutete das Ende der Konfessionalisierung der Prager Universität. Die Universität wurde eine eindeutig kalixtinische Lehranstalt. Außer dem Ausschluss von Katholiken erfolgte in jenem Jahr auch ein erster Eingriff gegen die Vertreter von Bruder Gregor, also gegen die Sympathisanten der zukünftigen Böhmischen Brüder unter den Prager Universitätsangehörigen. ${ }^{42}$ Die Prager Universität profilierte sich auf der Linie des offiziellen Utraquismus. Wer dem nicht zustimmen wollte, musste eine andere Hohe Schule wählen. ${ }^{43}$

39 MUP III, S. 51-52 (F. Šmahel - G. Silagi, Statuta, S. 55-56). In seiner Aufzeichnung macht der Rektor auch darauf aufmerksam, dass Hilarius dem König darüber geschrieben habe. Das Ziel, das Königreich zu vereinigen, formulierte Hilarius direkt in seinem Brief oder Traktat, der von Antonín PodLAHA, Hilaria Litoměřického List králi Jiř́mim [Das Schreiben des Hilarius von Leitmeritz an König Georg], Praha 1931 (Datierung in die zweite Hälfte von 1462) herausgegeben wurde. Über das Allerheiligenkapitel spricht er aber nicht. Dazu T. KaLIna, Václav Křržanonovský, S. 346-347 (das Blatt datiert er in die erste Hälfte von 1461); R. URBÁneK, Věk poděbradský IV, S. 245-246.

40 Jiří PЕŠEK, Některé otázky dějin univerzity pražské jagellonského obdobi (1471-1526) [Einige Fragen zur Geschichte der Prager Universität in der jagellonischen Periode (1471-1526)], AUC-HUCP 18/1, 1978, S. 129-171.

41 V. V. Tомек, Dějepis města Prahy, IX, S. 87-89; R. Urвánek, Věk poděbradský, IV, S. 243-244, 246; zur Idee der Rekatholisierung des Allerheiligenkapitels T. KaLina, Václav Křžănovský, S. 346-348.

42 Jaroslav Goll, Jednota bratrská v 15. století [Die Böhmischen Brüder im 15. Jahrhundert], III, První pronásledování bratř́ [Die erste Verfolgung der Brüder], Časopis musea Království českého 58, 1884, S. 447-471, hier S. 450-452. F. Šmahel, Počátky humanismu na pražské universitě, S. 63-64.

43 Zwei Beispiele des Auszugs polnischer Magister bei Robert T. TомсzAK, Polish professors at Prague universities (14th-18th centuries). A prosopographic study, hier in diesem Band. 
Unter den Magistern hinterließen diese Ereignisse gegenseitigen Hass, wie noch nach Jahren eine Bemerkung des Magisters Koranda an die Adresse jener bezeugt, die sich gegen seine Wahl zum Dekan gestellt hatten. ${ }^{44}$

Infolgedessen war der Wirkungskreis der Prager Universität im wesentlichen auf das utraquistische Gebiet Böhmens beschränkt. Infolge ihrer Konfessionalisierung wurde die Universität eine Landesuniversität.

Bei den untersuchten Ereignissen spielten königliche Eingriffe eine bedeutende Rolle. Bereits unter Wenzel IV. war das Treuegelöbnis auf den Herrscher eingeführt worden. Unter Georg von Podiebrad vertiefte sich der landesherrliche Charakter der Hohen Schule. ${ }^{45}$

Alle drei Prozesse - Konfessionalisierung, Territorialisierung und Etatisierung der Prager Universität - nahmen eine ähnliche Richtung in anderen Ländern voraus. Sie verfolgten ähnliche Tendenzen, die sich später in ganz Mitteleuropa zeigen sollten.

Mit dieser Entwicklung wurde zugleich die Chance vertan, in Prag alle Fakultäten zu erneuern. Aus dem Kollegium ausgeschlossen wurden der Theologe Křižanovský, der Jurist Hilarius und der Mediziner Krčín. Rokycanas systematische Bemühung, utraquistische Gelehrte und Theologen heranzuziehen und der Universität zu nützen, verfehlte somit ihre Wirkung.

Gesagt sei noch, dass ein Jahr später, im März 1462, alle zehn Magistranden gelobten, sich an die heiligen Wahrheiten zu halten, besonders an die Kommunion unter beiderlei Gestalt. ${ }^{46}$ Die Erklärung Papst Pius' II., die Kompaktaten hätten bereits ihre Gültigkeit verloren, erfolgte erst mehrere Tage danach, nämlich Ende März in Rom. ${ }^{47}$ Die Utraquisierung der Universität war also nicht die Folge der Entwicklung in Böhmen nach Aufhebung der Kompaktaten, sondern verlief Monate zuvor als interner Prozess der Prager Hohen Schule.

(C) Deutsche Übersetzung: Wolf B. Oerter

44 Neben ausgiebiger Polemik gegen Hilarius von Leitmeritz und einer polemischen Antwort auf die Schrift des Stanislaus von Welwarn ist dies eine giftige Bemerkung, die er in einem Brief an Ondřej Kuliš aus dem Jahre 1467 macht, indem er auf die drei Akteure der Universitätsaffäre anspielt: „,...tu insanis, qualiter apostatantibus uti Hilarius Vopice, Wenceslao Křivonosice et Girdoni Kurvice...". Zu Verweisen und zur Analyse siehe J. MAreK, Václav Koranda mladší, S. 61-67, 75-77, und S. 215-216, Nr. 8 und 11. Marek meint, Gira sei Georg von Meziříčí, es könnte aber wohl eher Georg aus der Prager Altstadt gemeint sein (s. Anm. 25). Weitere Äußerungen der Kritik bezüglich des Abweichlertums bei R. URBÁNEK, Věk podébradský IV, S. 248-249.

45 In den Statuten wird darauf direkt in den Marginalien mit der Bemerkung „Wenzels 1409“ hingewiesen. Vgl. auch MUP I/2, S. 75-76. Was das Kuttenberger Dekret in Gang gesetzt hatte, wird jetzt zur Norm. Vgl. Martin Nodu, Dekret kutnohorský, Praha 2010, S. 287-288 und Anm. 331 zu Kap. V (S. 403): ,...promittet fidelitatem regi nostro et regni“" (Statutenbuch, die Nationalbibliothek Prag, Handschrift XIV D 25, fol. 6v). Vgl. F. ŠMAHEL, Nejstarši statuta Univerzity Karlovy, S. XXXVI.

46 MUP I/2, S. 78: „Praescripti magistrandi sponte promiserunt veritatibus sacris usque etiam mortem firmiter adhaerere et specialiter communionem utriusque speciei sacratissimam ad vulgus protegere, et secundum posse defendere, confirmantes hoc manuum suarum per stipulationem...". V. V. TOMEK, Déjepis města Prahy, IX, S. 191-192; T. Kalina, Václav Křžanovský, S. 349, Anm. 2; R. URBÁneK, Věk podébradský, IV, S. 249 (Anm. 50 mit Beleg für den kleinen Auszug der katholischen Studenten); E. Gregorovičová, S. 112, Anm. 34. Kalina zufolge leisteten sie den Schwur auf das neue Statut der Vier Artikel freiwillig und es gebe keine Nachricht darüber, dass der König eingegriffen hätte (wie in der Literatur tradiert wird). Die Vier Artikel können sowohl den Universitätsbeschluss von 1458 als auch die eigentlichen Kompaktaten meinen.

47 František Šmahel, Die Basler Kompaktaten mit den Hussiten (1436). Untersuchung und Edition, Wiesbaden 2019, zur Außerkraftsetzung des Dokuments durch den Papst S. 105-108. 


\section{Finanzielle Förderung:}

Der Aufsatz entstand im Rahmen des Projekts Nr. GA18-00408S „Universitas magistrorum. Professoren der Prager utraquistischen Universität (1458-1622)“ der Tschechischen Agentur zur Förderung der wissenschaftlichen Forschung (Grantová agentura České republiky).

\section{ZAVRŠENÍ KONFESIONALIZACE PRAŽSKÉ UNIVERZITY NA PŘELOMU 50. A 60. LET 15. STOLETÍ NA PŘíKLADU OSUDU AKTIVNÍCH KATOLICKÝCH MISTRŮ}

\section{RESUMÉ}

Př́íspěvek se věnuje skupině mistrů vázaných na katolického administrátora Hilaria Litoměřického, kteří se pokusili ovládnout univerzitu koncem 50 . let 15 . století. Jedním z prostředků mělo být obnovené spojení kapituly a koleje Všech svatých pod pravomocí katolického probošta a administrátora. Na metropolitní kapitulu, resp. administrátory měla být stažena část soudních pravomocí rektora. Postupné odstranění katolické opozice z řad př́islušníků pražské univerzity za podpory českého krále zakončilo hledání profilu pražské školy. Byl završen proces konfesionalizace pražské univerzity. Vyrovnala se i se stoupenci Jednoty bratrské a od roku 1462 působila jako vyhraněně utrakvistická instituce provázaná s církví podobojí. V důsledku toho se zúžila spádová oblast pražské univerzity na utrakvistické území Čech. Utrakvizace školy z ní učinila univerzitu zemskou. Všechny tři procesy - konfesionalizace, teritorializace a etatizace pražské univerzity - předjímaly podobné vývojové trendy, které se prosadily později i v ostatních zemích střední Evropy.

PhDr. Blanka Zilynská, Ph.D.

Ústav dějin a archiv Univerzity Karlovy

blanka.zilynska@ruk.cuni.cz 